\title{
Environmental transmission electron microscopy using a conventional TEM and a gas injection-specimen heating holder
}

\author{
T. Kamino*, T.Yaguchi*, A.Watabe*, H.Saka** and K.Kishita*** \\ * Hitachi High-Technologies Corp., 11-1 Ishikawa-cho, Hitachinaka, Ibaraki, 312-0057 Japan \\ **Nagoya University, Furo-cho, Chikusa-ku, Nagoya, 4642-8603 Japan \\ *** Material Analysis Dept. Toyota Motors Corp., 1 Toyota-cho, Toyota 471-8572 Japan
}

In response to the increasing demands from material science, we have developed a gas injection specimen heating holder which allows in-situ observation of gas reacted materials at near atomic resolution at very high temperatures in a conventional TEM. The gas injection nozzle was built in the specimen heating holder and a spirally wound tungsten wire of 25 micrometer in diameter was used as the heating element[1-2]. Specimens sized in the order of micrometers are directly mounted on the heating element and nanometer sized specimens are distributed on a carbon coated heating element. The microscope used in the study was a Hitachi H-9500 300kV high resolution TEM. Spherical aberration co-efficient (Cs) and chromatic aberration co-efficient (Cc) of the microscope are $0.6 \mathrm{~mm}$ and $1.4 \mathrm{~mm}$, respectively. Since the specimen holder was designed not to give any interference to TEM image even at very high temperatures, this performance is thoroughly exploited for high resolution TEM observation of structural changes of specimens during gas reaction. AMT ERB high resolution digital imaging CCD camera system has been attached to the TEM and used for recording TEM images of dynamic behavior of the specimens. The specimen area and the electron gun area of the microscope are constantly evacuated by a 3601/s turbo molecular pump and a 601/s ion pump, respectively, and the two regions are separated by a $0.3 \mathrm{~mm}$ diameter fixed aperture located at the middle of the condenser system of the microscope. Thanks to these advanced vacuum technologies, the pressure of the electron gun chamber could be kept in the range of $10^{-5} \mathrm{~Pa}$ while the pressure at the specimen area was maintained at the range of $10^{-1} \mathrm{~Pa}$. This allows observation of high resolution TEM images using a $\mathrm{LaB}_{6}$ cathode at the accelerating voltage of $300 \mathrm{kV}$. The specimen heating holder with a gas injection nozzle and a carbon film coated heating element are shown in Fig. 1 a and b, respectively. The gas injection specimen heating holder is shown in Fig. 1. Pressures in the specimen chamber and the electron gun of the microscope as a function of the gas flow rate of the specimen heating holder is shown in Fig. 2. Example of dynamic HREM images observation of a growth of cubic- $\mathrm{In}_{2} \mathrm{O}_{3}$ at $373 \mathrm{~K}$ in the air atmosphere of $4.0 \times 10^{-1} \mathrm{~Pa}$ is shown in Fig.3. The lattice fringes of $\mathrm{In}_{2} \mathrm{O}_{3}(440)$ with the distance of $0.179 \mathrm{~nm}$ are clearly obtained in the video images.

\section{References}

[1] T. Kamino and H.Saka., Microanal. Microstruct. 4 (1993) 127-135

[2] T. Kamino et al., Mater. Trans. JIM 6 (1995) 73-75 


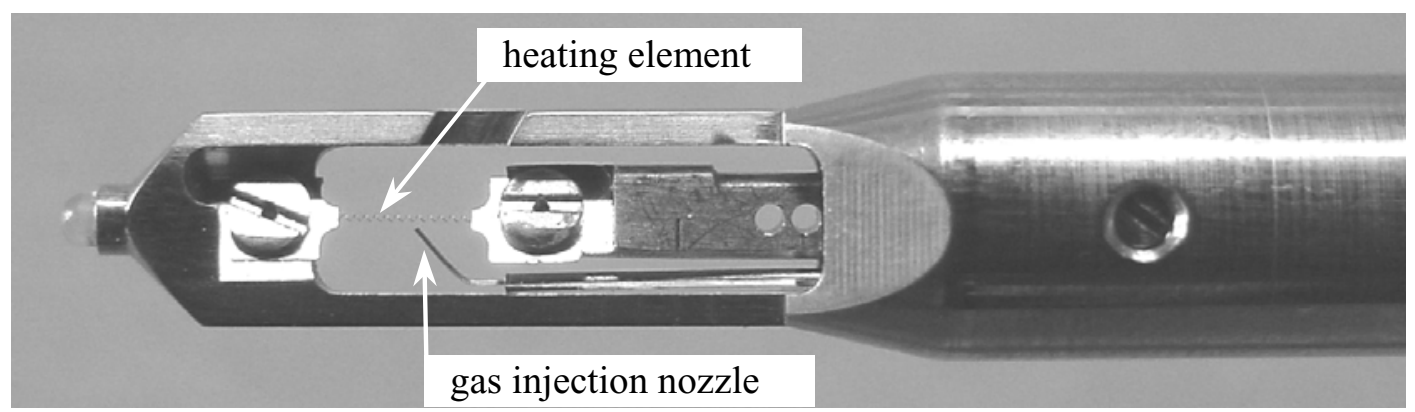

Fig.1 A gas injection-specimen heating holder developed for use with conventional TEMs.

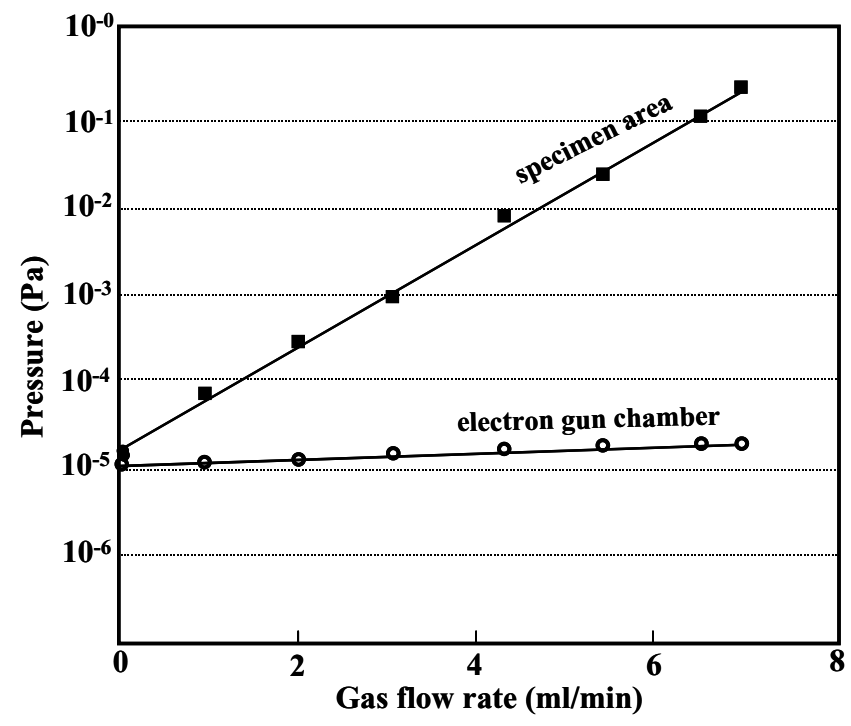

Fig.2 Pressures in the specimen area and the electron gun chamber as a function of the gas flow rate.

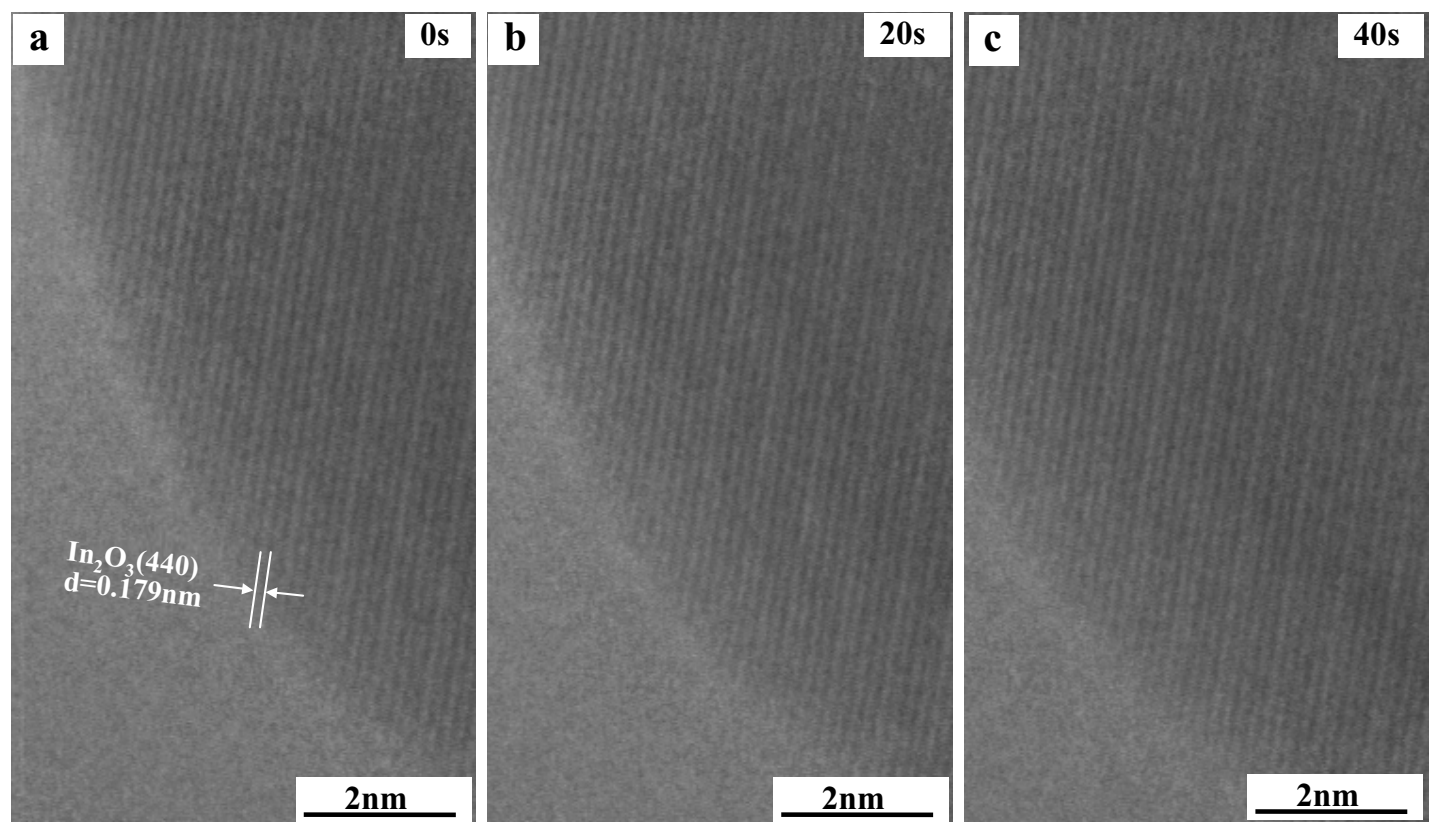

Fig.3 Series of HREM images of a growing cubic- $\operatorname{In}_{2} \mathrm{O}_{3}$ crystal observed at $373 \mathrm{~K}$ in the air atmosphere of $4.0 \times 10^{-1} \mathrm{~Pa}$. 